\title{
Active Transonic Aerofoil Design Optimization Using Robust Multiobjective Evolutionary Algorithms
}

\author{
D. S. Lee, ${ }^{*}$ J. Periaux, ${ }^{\dagger}$ and E. Onate \\ International Center for Numerical Methods in Engineering, 08034 Barcelona, Spain \\ L. F. Gonzalez ${ }^{\S}$ \\ Australian Research Centre Aerospace Automation, Queensland University of Technology, \\ Queensland 4009, Australia \\ and \\ N. Qin \\ University of Sheffield, Sheffield, England S10 2TN, United Kingdom \\ DOI: 10.2514/1.C031237
}

\begin{abstract}
The use of adaptive wing/aerofoil designs is being considered, as they are promising techniques in aeronautic/ aerospace since they can reduce aircraft emissions and improve aerodynamic performance of manned or unmanned aircraft. This paper investigates the robust design and optimization for one type of adaptive techniques: active flow control bump at transonic flow conditions on a natural laminar flow aerofoil. The concept of using shock control bump is to control supersonic flow on the suction/pressure side of natural laminar flow aerofoil that leads to delaying shock occurrence (weakening its strength) or boundary-layer separation. Such an active flow control technique reduces total drag at transonic speeds due to reduction of wave drag. The location of boundary-layer transition can influence the position and structure of the supersonic shock on the suction/pressure side of aerofoil. The boundarylayer transition position is considered as an uncertainty design parameter in aerodynamic design due to the many factors, such as surface contamination or surface erosion. This paper studies the shock-control-bump shape design optimization using robust evolutionary algorithms with uncertainty in boundary-layer transition locations. The optimization method is based on a canonical evolution strategy and incorporates the concepts of hierarchical topology, parallel computing, and asynchronous evaluation. Two test cases are conducted: the first test assumes the boundary-layer transition position is at $\mathbf{4 5 \%}$ of chord from the leading edge, and the second test considers robust design optimization for the shock control bump at the variability of boundary-layer transition positions. The numerical result shows that the optimization method coupled to uncertainty design techniques produces Pareto optimal shock-control-bump shapes, which have low sensitivity and high aerodynamic performance while having significant total drag reduction.
\end{abstract}

\section{Nomenclature}

$c$
$C_{l}$
$C_{p}$
$C d_{\text {Total }}$
$C d_{\text {Viscous }}$
$C d_{\text {Wave }}$
$f$
$K$
$R e$
$\operatorname{SCB}_{H}$
$\operatorname{SCB}_{L}$
$\operatorname{SCB}_{P}$
$\alpha$

$=$ chord

$=$ lift coefficient

$=$ pressure coefficient

$=$ viscous drag coefficient

$=$ wave drag coefficient

fitness functions

$=$ Reynolds number

$=$ angle of attack
$=$ total coefficient, $C d_{\text {Viscous }}+C d_{\text {Wave }}$

number of uncertainty step size

$=$ height of shock control bump $(\% c)$

$=$ length of shock control bump $(\% c)$

$=$ peak position of shock control bump $\left(\% \mathrm{SCB}_{L}\right)$
Presented as Paper at the 49th AIAA Aerospace Sciences Meeting, Orlando, FL, 4-7 January 2011; received 14 September 2010; revision received 11 November 2010; accepted for publication 18 January 2011. Copyright $(2011$ by the American Institute of Aeronautics and Astronautics, Inc. All rights reserved. Copies of this paper may be made for personal or internal use, on condition that the copier pay the $\$ 10.00$ per-copy fee to the Copyright Clearance Center, Inc., 222 Rosewood Drive, Danvers, MA 01923; include the code 0021-8669/11 and \$10.00 in correspondence with the CCC.

*Senior Scientist CIMNE. Member AIAA.

${ }^{\dagger}$ Professor, CIMNE/UPC. Associate Fellow AIAA.

†Professor, CIMNE/UPC

${ }^{\S}$ Lecturer, ARCAA School of Engineering System. Member AIAA.

TProfessor, Department of Mechanical Engineering. Associate Fellow AIAA.

\section{Introduction}

T ENGINEERING designs, it is inevitable to face uncertainty design parameters that must be considered in an optimization task to produce a set of reliable optimal solutions (high performance and low sensitivity). Some of the design variables and system input parameters cannot be achieved exactly due to uncertainties in physical quantities such as manufacturing tolerances, material properties, and environmental conditions, including temperature, pressure, velocity, etc. In conventional design, these parameters are treated as a constant value with assumption. The design model obtained by conventional design methods has good performance at the standard design point; however, using fixed/constant values for uncertain parameters makes a design model fluctuate at offdesign conditions. In other words, the design model has high sensitivity at a set of unstable conditions that produces an unstable performance $[1,2]$. An alternative design strategy is the use of robust design when considering uncertainty design parameters to control the sensitivity and performance of the model [3,4]. In this paper, an active flow control (AFC) device design is considered as a robust optimization application.

One of the important challenges in aeronautical engineering is to control flow over the aerofoil/wing to reduce drag while increasing lift. One of the main reasons is that the drag reduction can save mission operating costs, condense critical aircraft emissions, and increase the performance envelope of the aircraft. Such drag reduction can be achieved by implementing a type of AFC called shock control bump (SCB) [5-8] without the need to design a new aerofoil or wing planform shape.

In this paper, a natural laminar flow (NLF) aerofoil, the Royal Aircraft Establishment (RAE) 5243 (used as a baseline design), is investigated with SCB on the suction side to reduce total drag and to 
extend its critical Mach and lift coefficients at high lift coefficient and transonic flow speeds. Two optimization test cases are conducted using advanced evolutionary algorithms (EAs) [9], and the first test considers a boundary-layer transition (BLT) (note that, in aerodynamics, BLT is often used as $x_{\mathrm{tr}}$ ) position at $45 \%$ of chord. The second test is conducted with uncertainty design parameters, the variability of BLT positions, and lift coefficient values using a robust/ uncertainty design technique. In conventional design, aerofoil optimization is conducted at a fixed/constant BLT position. However, BLT cannot be assumed as a constant value in a real design problem, especially for the AFC device design since the shape of AFC device will change the position of BLT. Therefore, the BLT location is considered as one of the uncertainty design parameters.

The paper will show how to control the design quality while considering uncertain design parameters, and it will demonstrate how to control the transonic flow on the current aerofoil using a SCB.

The rest of the paper is organized as follows. Section II describes an optimization method and uncertainty design techniques. Aerodynamic analysis tools are described in Sec. III. Section IV demonstrates the use of SCB. Section V considers SCB shape design optimization using single-objective and robust design approaches considering uncertain BLT positions. The overall results obtained in Sec. V are discussed in Sec. VI. The paper concludes with a summary and some future research avenues in Sec. VII.

\section{Robust/Uncertainty Design Methods}

The method couples hierarchical asynchronous parallel multiobjective EAs (HAPMOEAs) with an algorithm for SCB geometry, a module for robust design, and several aerodynamic analysis tools [9].

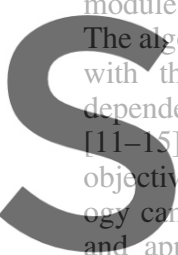
orithm is based on evolution concepts of covariance , and Pareto tournament selec e or multiobjective problem: provide different models, include
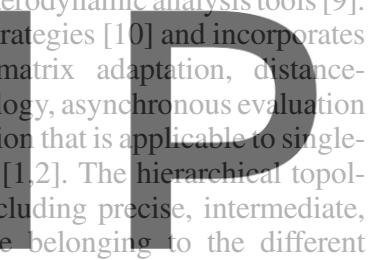
hierarchical layer can be handled by a different EA code, HAPMQEA has also been coupled to uncertainty design techniques,

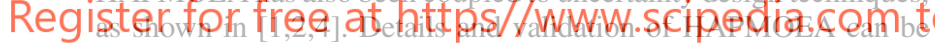 found in [9].}

\section{A. Robust/Uncertainty Design}

A robust design method, also called the Taguchi method (uncertainty) [3], improves the quality of engineering productivity. An optimization problem can be defined as follows:

maximization or minimization

$$
f=f\left(x_{1}, \ldots, x_{n}, x_{n+1}, \ldots, x_{m}\right)
$$

where $x_{1}, \ldots, x_{n}$ represent design parameters and $x_{n+1}, \ldots, x_{m}$ represent uncertainty parameters. The range of uncertainty design parameters can be defined by using two statistical functions: mean $\bar{x}$ and variance $\left[\delta x=(\sigma x)^{2}\right]$ as part of the PDF.

The Taguchi optimization method minimizes the variability of the performance under uncertain operating conditions. Therefore, in order to perform an optimization with uncertainties, the fitness function(s) should be associated with two statistical formulas: the mean value $\bar{f}$ and its variance $\delta f$ or standard deviation $\sigma f=\sqrt{\delta f}$ :

$$
\begin{gathered}
\bar{f}=\frac{1}{K} \sum_{i=1}^{K} f_{i} \\
\delta f=\frac{1}{K-1}\left[\sum_{i=1}^{K}\left(f_{i}-\bar{f}\right)^{2}\right]
\end{gathered}
$$

where $K$ denotes the number of subintervals of variation flow conditions.

The values obtained by the mean $\bar{f}$ and the variance $\sigma f$ or standard deviation $\delta f$ represent the reliability of the model in terms of the magnitude of performance and stability/sensitivity at a set of uncertain design conditions.

\section{B. Robust Design Method Implementation for Single-Objective Design Optimization Problems}

The problem definition can be written with the fitness functions associated with a mean [Eq. (2)] and variance/standard deviation [Eq. (3)] as an uncertainty-based multiobjective design problem if a single-objective aerofoil design optimization problem considers a minimization of drag $\left[f=\min \left(C_{D}\right)\right]$ at a single flight condition $M_{S}$. The robust design fitness functions are shown in Eqs. (4) and (5), while a single flight condition becomes a set of uncertainty flight conditions; $M_{\infty_{K}} \in\left[M_{S}-\varepsilon, M_{S}, M_{S}+\varepsilon\right]$ or $\bar{M}_{\infty}$ and $\sigma M_{\infty}$ :

$$
\begin{gathered}
\bar{C}_{D}=\frac{1}{K} \sum_{i=1}^{K} C_{D_{i}} \\
\sigma C_{D}=\sqrt{\delta C_{D}}=\sqrt{\frac{1}{K-1}\left[\sum_{i=1}^{K}\left(C_{D_{i}}-\bar{C}_{D}\right)^{2}\right]}
\end{gathered}
$$

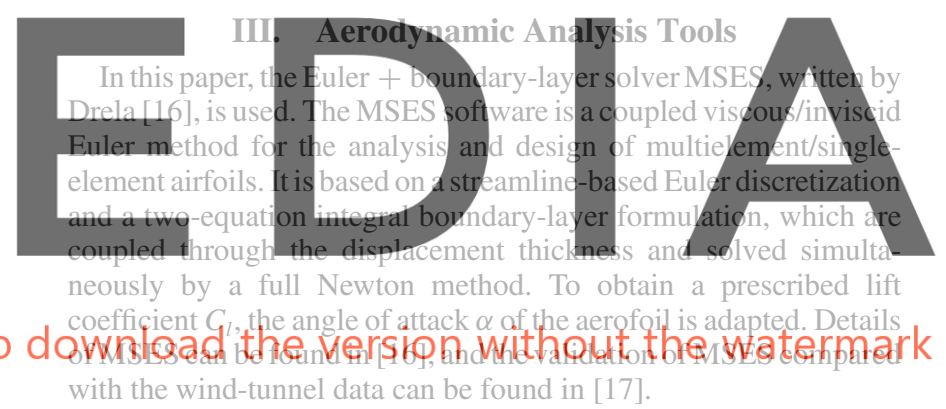

\section{Wave Drag Reduction via Active Flow Control Bump}

At transonic speed, the flow over the high camber wing causes shock waves where there is a large change of gas properties and the flow becomes irreversible. Through the shock, total pressure decreases and entropy increases, which means an increase in the wave drag. Ashill et al. [5] proposed the concept of a transonic bump using geometry adaptation on an aerofoil to control shock behavior that is also called SCB. As illustrated in Fig. 1, the most common design variables for the $\mathrm{SCB}$ are length $\mathrm{SCB}_{L}$, height $\mathrm{SCB}_{H}$, and peak position $\mathrm{SCB}_{P}$, and the center of the $\mathrm{SCB}$ (i.e., $50 \%$ of $\mathrm{SCB}_{L}$ ) is located where the shock occurs on the transonic aerofoil design.

Figure 2 illustrates the concept and benefit of using SCB. The transonic flow over normal aerofoil without SCB accelerates to supersonic, and the pressure forms a strong shock that leads a high $C d_{\text {Wave }}$; however, the pressure difference over the SCB causes the

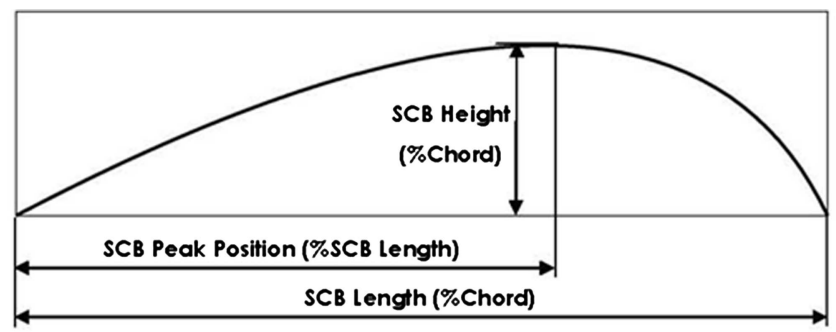

Fig. 1 Design components of SCB. 


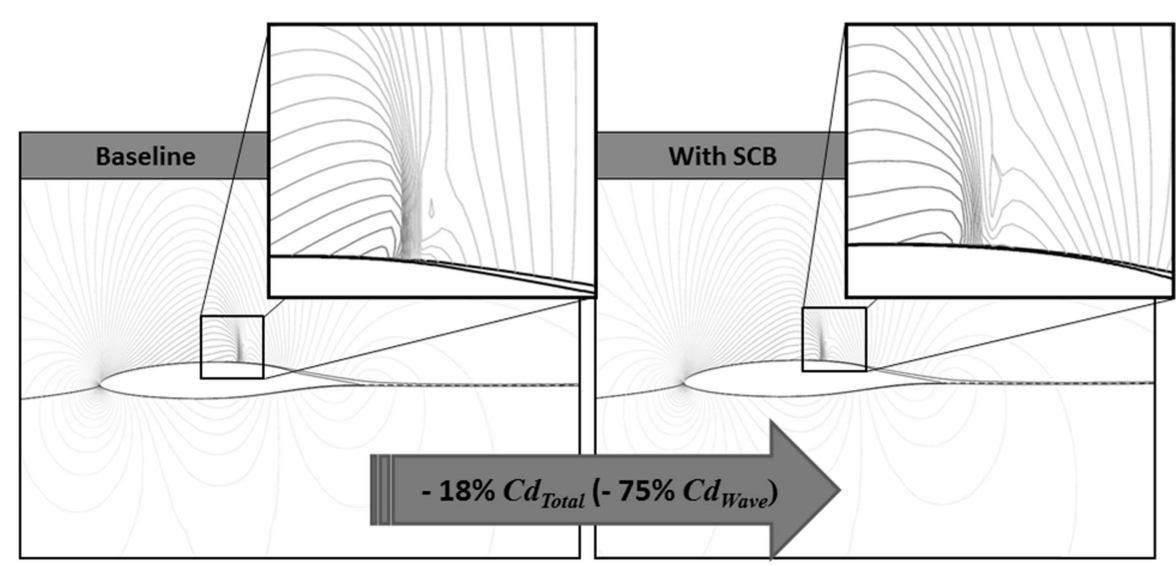

Fig. 2 Comparison of aerofoil without (left) and with SCB (right).

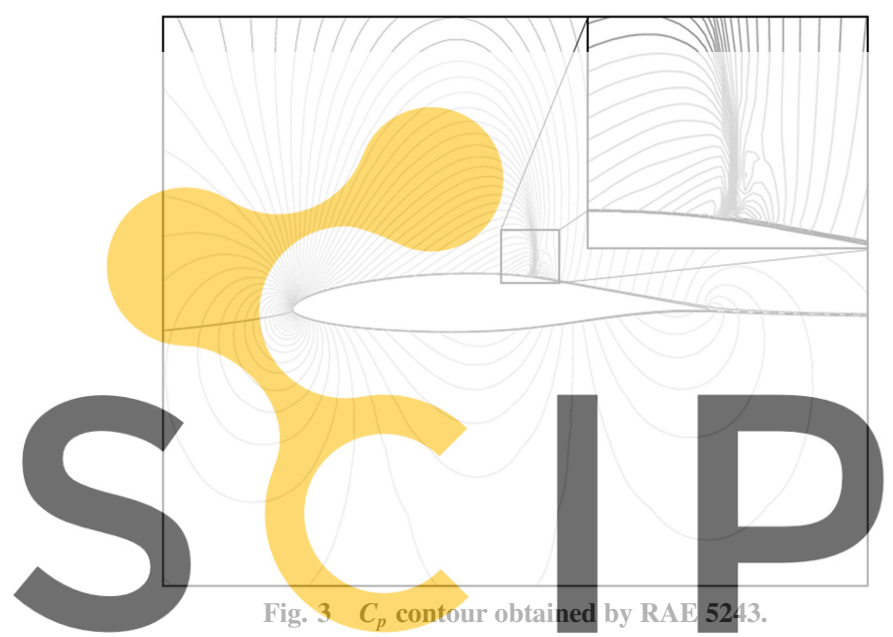

Regisupersonic flow to decelerate to subsonic Mach numbers by a weake

\section{Active Flow Control Bump (Shock Control Bump) Design Optimization}

The NLF aerofoil, RAE 5243 [6], is selected as a baseline design. The aerofoil has a maximum thickness of 0.14 at $41 \% c$ from the leading edge and a maximum camber of 0.0186 at $54.0 \% \mathrm{c}$. The baseline design is tested at flow conditions $M_{\infty}=0.68, C_{l}=0.82$, and $R e=19.0 \times 10^{6}$, with a BLT position at $45 \%$ of chord from the leading edge. Figure 3 shows the $C_{p}$ contours obtained by MSES. It can be seen that there is a strong normal shock on the suction side of the baseline design at $45 \% c$ BLT position.

The shock occurs at $59.5 \%$ of chord, where a BLT position is at $45 \% c$. This baseline design will be compared with the optimal SCB designed to minimize the total drag $C d_{\text {Total }}$ in Secs. V.A and V.B.

A. Shock-Control-Bump Shape Design Optimization with Boundary-Layer Transition Position at $\mathbf{4 5 \%}$ c

1. Problem Definition

This test case considers a single-objective SCB design optimization on the upper surface of the RAE 5243 aerofoil to minimize the total drag at flow conditions $M_{\infty}=0.68, C_{l}=0.82$, and $R e=$

Table 1 SCB design variables and bounds

\begin{tabular}{lcc}
\hline \hline Design variables & Lower bound & Upper bound \\
\hline $\mathrm{SCB}_{L}$ & 0 & 30 \\
$\mathrm{SCB}_{H}$ & 0 & 5 \\
$\mathrm{SCB}_{P}$ & 0 & 100 \\
\hline \hline
\end{tabular}

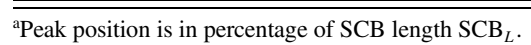

$19.0 \times 10^{6}$ with BLT position at $45 \% c$ from the leading edge. The fitness function is shown in Eq. (6):

$$
\text { fitness }(f)=\min \left(C d_{\text {Total }}\right)=\min \left(C d_{\text {Viscous }}+C d_{\text {Wave }}\right)
$$

\section{Design Variables}

The design variable bounds for the SCB geometry are illustrated in Table 1. The center of the SCB (50\% of SCB length) will be located at the shock where the flow speed transits from supersonic to subsonic (60\% of chord in this case). The SCB spans from approximately $45 \%$ of chord to $75 \%$ of chord if the SCB length is $30 \%$ of chord.

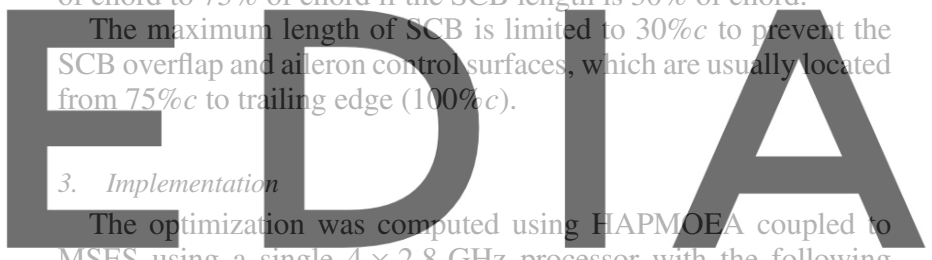
MSES using a single $4 \times 2.8 \mathrm{GHz}$ processor with the following details on multiresolution/population hierarchical populations [7]:

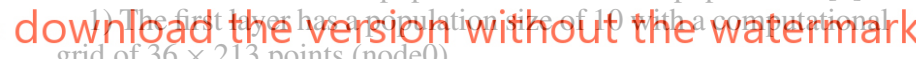

2) The second layer has a population size of 20 with a computational grid of $24 \times 131$ points (node1 and node2).

3) The third layer has a population size of 30 with a computational grid of $36 \times 111$ points (node $3 \sim$ node 6 ).

Note that the difference in accuracy between the first and the third layers is less than $5 \%$.

\section{Numerical Results}

The algorithm was allowed to run for $24 \mathrm{~h}$ (as time stopping criterion) and for 6135 function evaluations. Convergence occurred after 1826 function evaluations (7.6 h), as shown in Fig. 4.

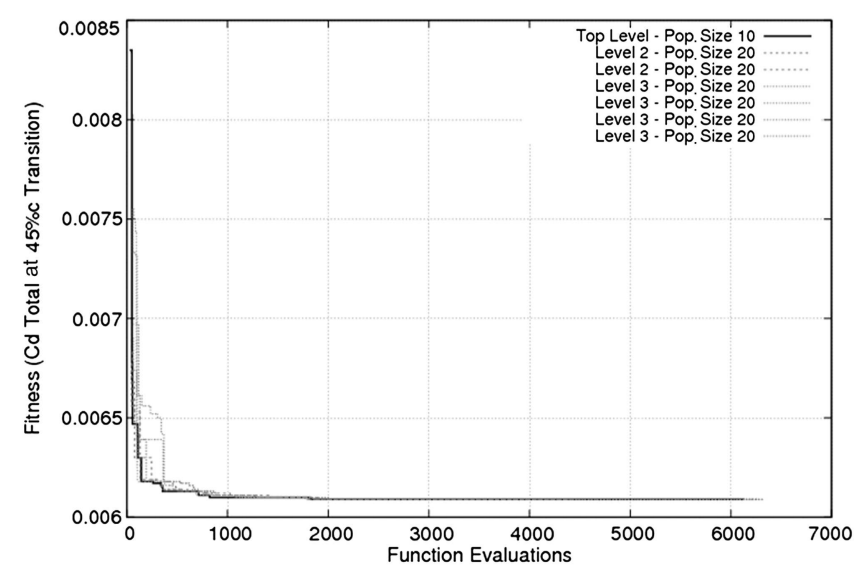

Fig. 4 Convergence objective for SCB design at $45 \% c$ BLT. 
Table 2 Aerodynamic characteristics obtained by single-objective design approach

\begin{tabular}{lccc}
\hline \hline Aerofoil & $C d_{\text {Total }}$ & $C d_{\text {Wave }}$ & $L / D$ \\
\hline Baseline (RAE 5243) & 0.01003 & 0.0032 & 81.72 \\
with optimal SCB & $0.00609(-40 \%)$ & $0.00014(-95 \%)$ & $134.56(+65 \%)$ \\
\hline \hline
\end{tabular}

${ }^{\mathrm{a}} C_{l}$ is fixed to 0.82 .

Table 3 Optimal SCB design components

\begin{tabular}{lc}
\hline \hline Variables & $\mathrm{SCB}$ \\
\hline $\mathrm{SCB}_{L}, \% c$ & 29.22 \\
$\mathrm{SCB}_{H}, \% c$ & 1.04 \\
$\mathrm{SCB}_{P}\left(\% \mathrm{SCB}_{L}\right)$ & 67.7 \\
\hline \hline
\end{tabular}

aPeak position $\mathrm{SCB}_{P}$ is in percentage of SCB length; and the optimal SCB is located between $x=0.4516$, $y=0.0858$ and $x=0.7440, y=0.0475$.

Table 2 compares the aerodynamic characteristics obtained by the baseline design (RAE 5243) and the baseline design with upper SCB. It can be seen that applying SCB on the upper surface of an RAE 5243 aerofoil reduces the wave drag by $95 \%$, which leads to $40 \%$ of the total drag reduction. This optimal SCB improves $L / D$ by $65 \%$.

The design variables for the optimal SCB are shown in Table 3. Figure 5 compares the geometry of the baseline design and the baseline with optimal SCB. The baseline (RAE 5243) design with optimal SCB has a maximum thickness of $0.14\left(t / c_{\max }=0.14\right)$ at $4.1 \% \mathrm{c}$ from the leading edge, and the maximum camber is 0.0215 at $63.1 \% \mathrm{c}$. Adding the optimal SCB inc 0.003 , and the camber position move 9 \%oc while keeping the same maxin design.
Figu
with op e 6a shows the $C_{p}$ contour o timal SCB. It can be seen that the toward to
um thickn
tained by
we strong sh
weaker by

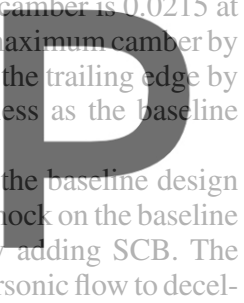
pressure difference over the SCB causes the supersonic flow to decelRegister for fre each numbers by a weaker shock wive, which if Figures $7 \mathrm{a}$ and $7 \mathrm{~b}$ compare total drag $C d_{\text {Total }}$ and wave drag $C d_{\text {Wave }}$ distributions obtained by the baseline design and with the optimal SCB along the Mach range; that is, $M_{\infty} \in[0.5: 0.75]$ with $C_{l \text { Fixed }}=0.82$, and $R e=19.0 \times 10^{6}$ with a BLT position at $45 \%$. The baseline design with SCB starts to produce lower total drag when the Mach number is higher than 0.67. By adding the optimal SCB, the baseline design reduces its total drag by $40 \%$ and its wave drag by $95 \%$ at the standard flight condition marked with the dashed line. The critical Mach number for baseline design $\left(M_{C}=0.65\right)$ is extended to 0.68 due to the optimal SCB.

Figures $8 \mathrm{a}$ and $8 \mathrm{~b}$ compare total drag distributions obtained by the baseline design and with the optimal SCB for a $C_{l}$ range; that is, $C_{l} \in$ [0.1:1.1] with $M_{\infty}=0.68$ and $R e=19.0 \times 10^{6}$ with BLT position at $45 \% c$. The baseline design with SCB starts to produce lower total drag when the $C_{l}$ number is higher than 0.65 . The critical $C_{l}$ number for the baseline design $\left(C_{l c}=0.5\right)$ is extended to 0.8 by applying optimal SCB on the suction side of baseline design.

Even though good results were obtained by using the singleobjective design approach at the standard flow conditions, the optimal SCB produces an irregular/undesirable $C d_{\text {Total }}$ fluctuation at a range of $C_{l}$ from 0.6 to 0.82 , as shown in Figs. $8 \mathrm{a}$ and $8 \mathrm{~b}$. This

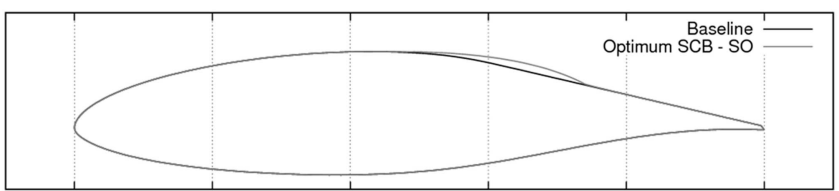

Fig. 5 Baseline design with optimal SCB at $45 \% c$ BLT (SO denotes single objective). optimal solution is an overoptimized solution that does not perform well before reaching the standard flow/flight conditions. Such a fluctuation should be treated as uncertainty in the design parameters, and the design engineer should take into account during the optimization. Therefore, it is necessary to use an uncertainty design technique to produce a set of solutions that have both low mean and sensitivity $C d_{\text {Total }}$ by considering variable $C_{l}$ values and BLT positions.

\section{B. Robust Shock-Control-Bump Shape Design Optimization with} Uncertainty in Boundary-Laver Transition Locations

1. Problem Definition

This test case considers a robust multiobjective SCB design optimization on the upper surface of the RAE 5243 aerofoil to minimize mean and standard deviation of total drag $\left(\overline{C d_{\text {Total }}}\right.$ and $\left.\sigma C d_{\text {Total }}\right)$ at the flight conditions $M_{\infty}=0.68$ and $R e=19.0 \times 10^{6}$. For uncertainty design parameters, two $C_{l}$ and three BLT positions are considered: that is, $C_{l}=[0.7,0.82]$ and $\mathrm{BLT}=$ $[25 \% \mathrm{c}, 37.5 \% \mathrm{c}, 50 \% \mathrm{c}]$. These can be statistically written as $\overline{\mathrm{BLT}}=37.5 \% \mathrm{c}$ and $\sigma \mathrm{BLT}=12.5 \% \mathrm{c}$. The candidate SCB model
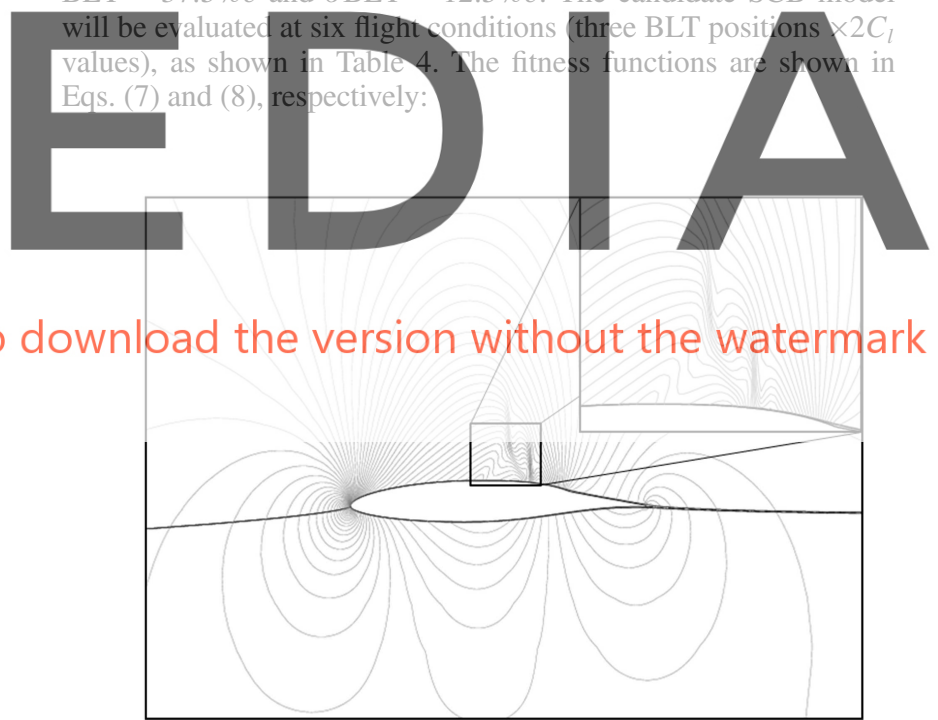

a)

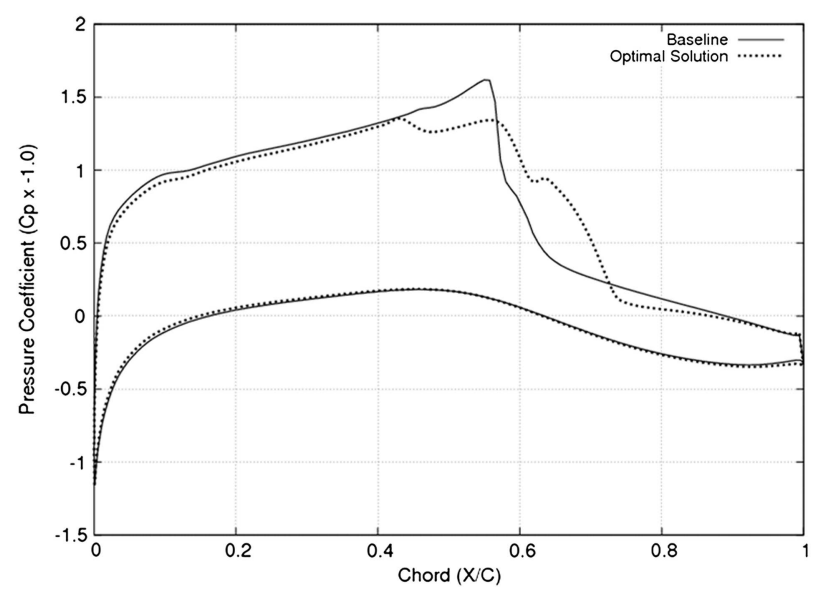

b)

Fig. $6 C_{p}$ contour: a) $C_{p}$ distribution and b) obtained by RAE 5243 with the optimal SCB at $45 \% c$ BLT position. 


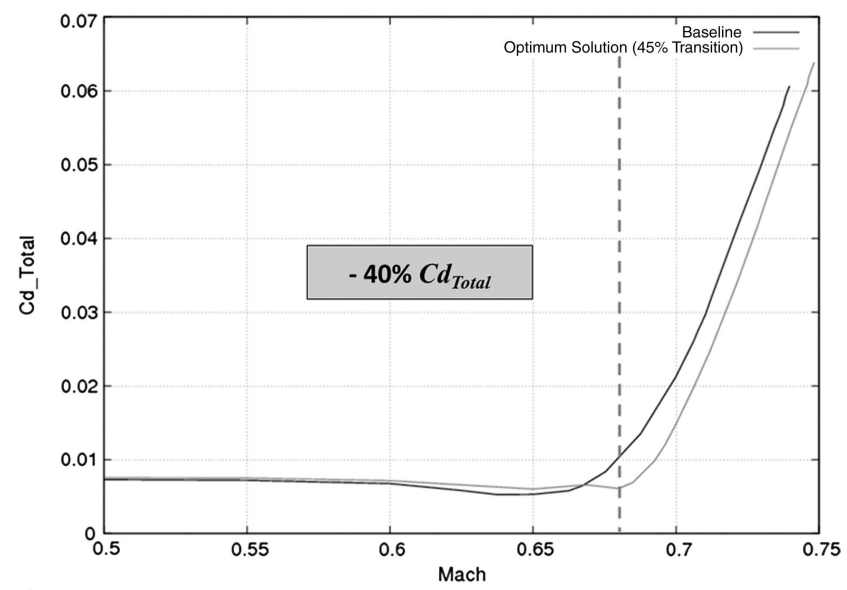

a)

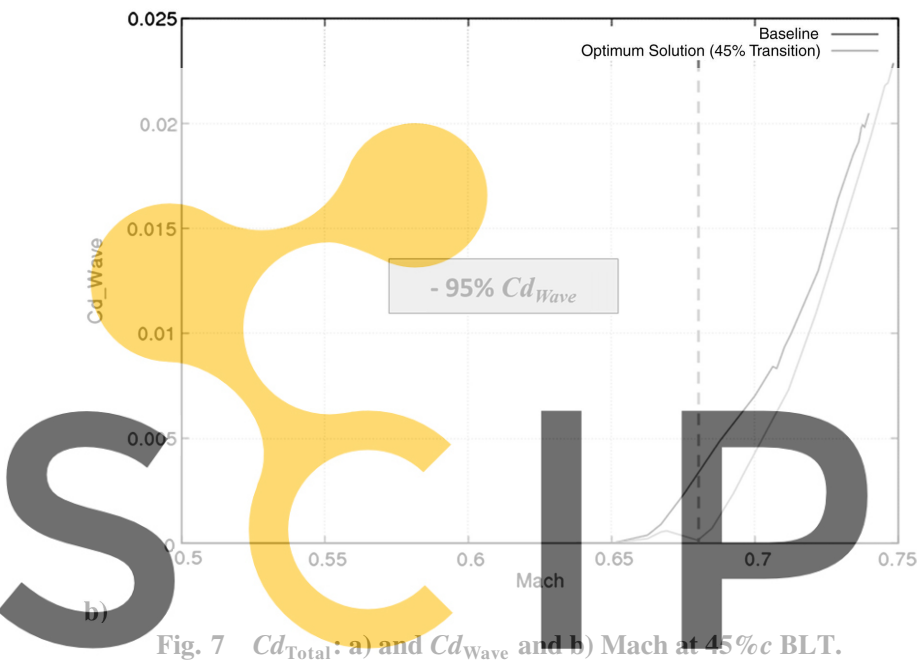

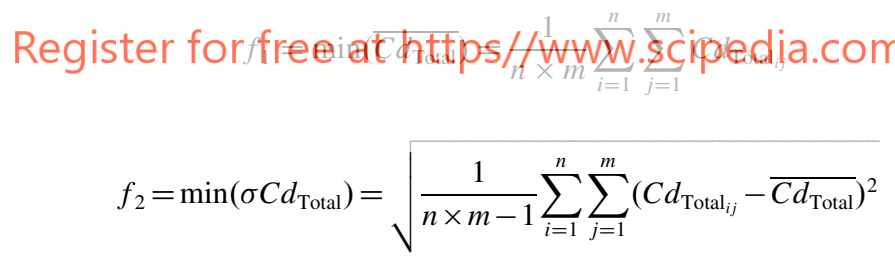

where $n$ and $m$ represent the number of BLT positions and $C_{l}$ conditions.

\section{Design Variables}

The design variable bounds for the SCB geometry were illustrated in Table 1 . The center of the SCB $\left(50 \%\right.$ of $\left.\mathrm{SCB}_{L}\right)$ will be located at the sonic point where the flow speed transits from supersonic to subsonic (58\% of chord) at $37.5 \% c$ BLT. The SCB will be located between $43 \% c$ to $73 \% c$ if SCB length is $30 \% c$.

\section{Implementation}

The optimization was computed using HAPMOEA coupled to MSES with the following details on multiresolution/population hierarchical populations [7]:

1) The first layer has a population size of 15 with a computational grid of $36 \times 213$ points (node 0 ).

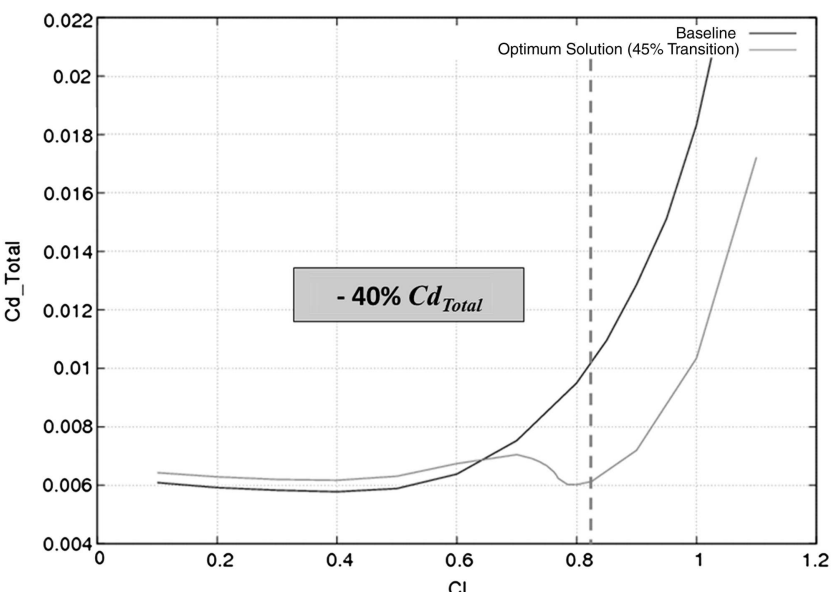

a)

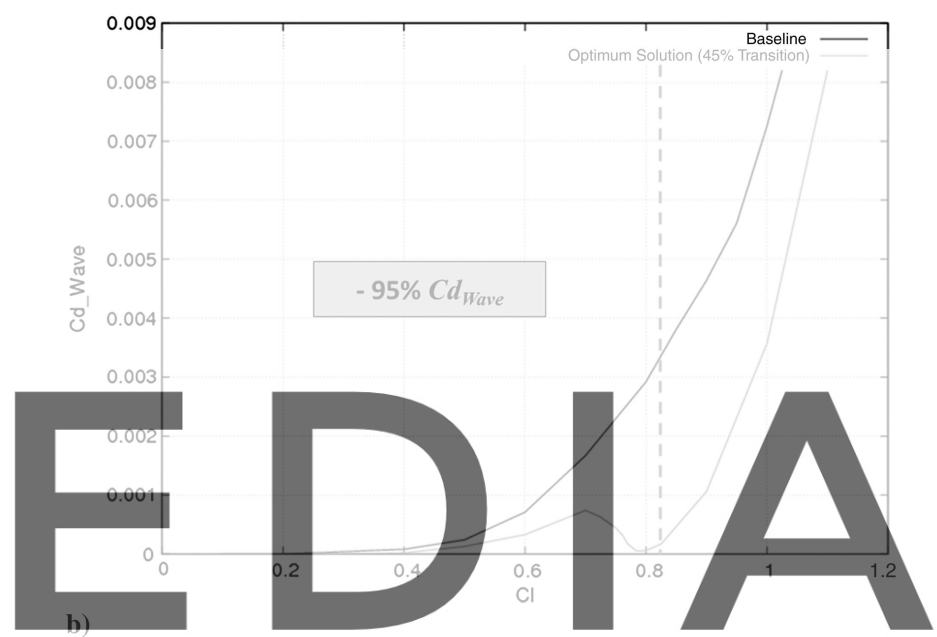

Fig. $8 C d_{\text {Total }}$ : a) $C d_{\text {Wave }}$ and b) $C_{l}$ at $45 \% c$ BLT.

download the version without the watermark

2) The second layer has a population size of 20 with a computational grid of $24 \times 131$ points (node 1 and node2).

3) The third layer has a population size of 40 with a computational grid of $36 \times 111$ points (node $3 \sim$ node6).

Note that the difference in accuracy between the first and third layers is less than $5 \%$.

\section{Numerical Results}

The algorithm was allowed to run for $50 \mathrm{~h}$ and 2450 function evaluations using a single $4 \times 2.8 \mathrm{GHz}$ processor. Pareto optimal solutions are shown in Fig. 9, and their sensitivity and performance are compared with the baseline design and the optimal design from the single-objective approach (Sec. V.A). It can be seen that all Pareto members dominate the baseline for the second fitness function (standard deviation of $C d_{\text {Total }}$ ), while Pareto members 1 to 9 dominate the baseline design in terms of mean and standard deviation of $C d_{\text {Total }}$. Pareto members 1 to 3 dominate the optimal design from Sec. V.A. Pareto members 1 and 3 are selected to compare aerodynamic performance with the baseline design and the optimal solution from Sec. V.A.

Table 5 compares the aerodynamic characteristics obtained by the baseline design (RAE 5243) and the baseline design with SCB obtained by Pareto members 1 and 3 . It can be seen that applying the

Table 4 Variability of flight conditions (three BLT positions $\times 2 C_{l}$ )

\begin{tabular}{ccccccc}
\hline \hline Variability & Flight condition 1 & Flight condition 2 & Flight condition 3 & Flight condition 4 & Flight condition 5 & Flight condition 6 \\
\hline BLT position, $\% c$ & 25.0 & 25.0 & 37.5 & 37.5 & 50.0 & 50.0 \\
$C_{l}$ & 0.7 & 0.82 & 0.7 & 0.82 & 0.7 & 0.82 \\
\hline \hline
\end{tabular}




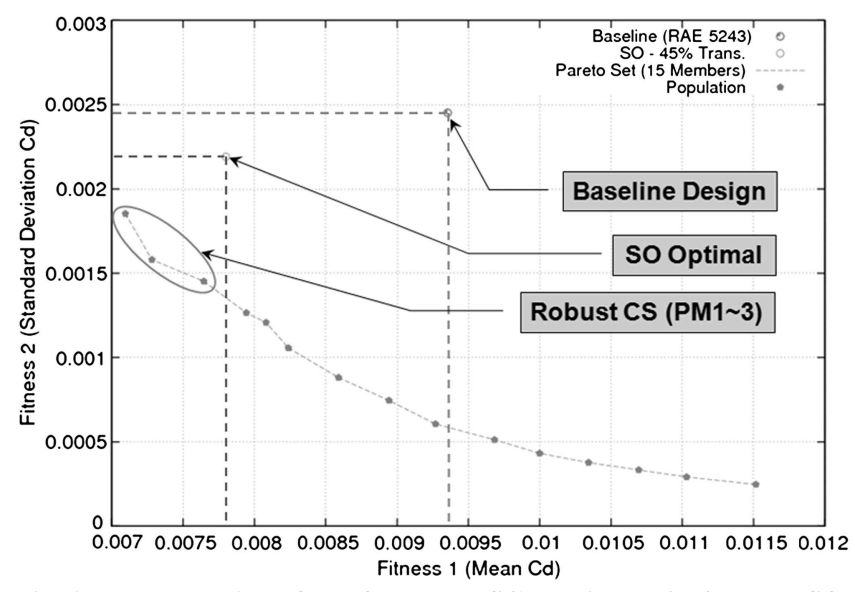

Fig. 9 Pareto optimal front for robust SCB design at 45\%c BLT. SO, CS, and PM represent the single-objective approach, compromised solutions, and Pareto members, respectively.

optimal SCB obtained by Pareto member 1 on the suction side of the RAE 5243 aerofoil reduces the mean total drag by $24 \%$. This optimal SCB improves $L / D$ by $32.0 \%$.

The mean and standard deviations obtained by the baseline design, single-objective, and robust Pareto members can be compared using cumulative the distribution function $(\mathrm{CDF})$ and the probability density function (PDF). Figure 10a shows the CDF obtained by the baseline design, the optimal from the single-objective design (marked as SO optimal in figure), and the robust compromise Pareto

Colutions (marked as robust CS). It can he see that all solutions obtained by single-objective and robus mean total drag when compared wi member 1 reduces the mean total drag by $24 \%$ when compared with the baseline, while the optimal obtained by the single-objective approach reduces total drag by $17 \%$. The standard deviation (sensilivity) can be represented by evaluating the gradient of the lines to the CDF value of 0.5 or 1 (steep gradient $=$ low sensitivity). The PDF is plotted in Fig. $10 \mathrm{~b}$ to have a clear sensitivity comparison

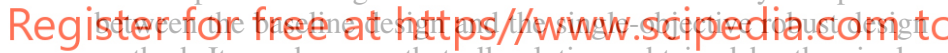
method. It can be seen that all solutions obtained by the singleobjective and robust design methods have lower sensitivity (narrower and taller bell curve). Pareto member 3 , obtained by the robust design method, has $41 \%$ total drag sensitivity reduction when compared with the baseline design, while the optimal obtained by the singleobjective approach reduces total drag by only $11 \%$. In other words, the robust design method has capabilities to produce a set of solutions that have better performance and sensitivity when compared with the single-objective optimization method.

For the detailed sensitivity analysis, the baseline design, the optimal solution obtained in Sec. V.A, and Pareto members 1 to 3 are selected and their $\overline{C d_{\text {Total }}}$ [Eq. (7)] and $\sigma C d_{\text {Total }}$ [Eq. (8)] are recomputed at the variability of $\overline{\mathrm{BLT}}=37.49 \% c, \sigma \mathrm{BLT}=$ $0.0729 \% c[25.0 \% c: 50.0 \% c]$, and the variability of $\bar{C}_{l}=0.761$, $\sigma C_{l}=0.0385$, and [0.7:0.82]. In total, 500 nonuniformly distributed flight conditions (50 BLT positions $\times 10 C_{l}$ values) obtained by Latin hypercube sampling [18] are considered. Table 6 compares the aerodynamic characteristics obtained by the baseline design, the optimal solution obtained in Sec. V.A, and Pareto members 1-3. It can be seen that both optimal solutions produce lower total mean

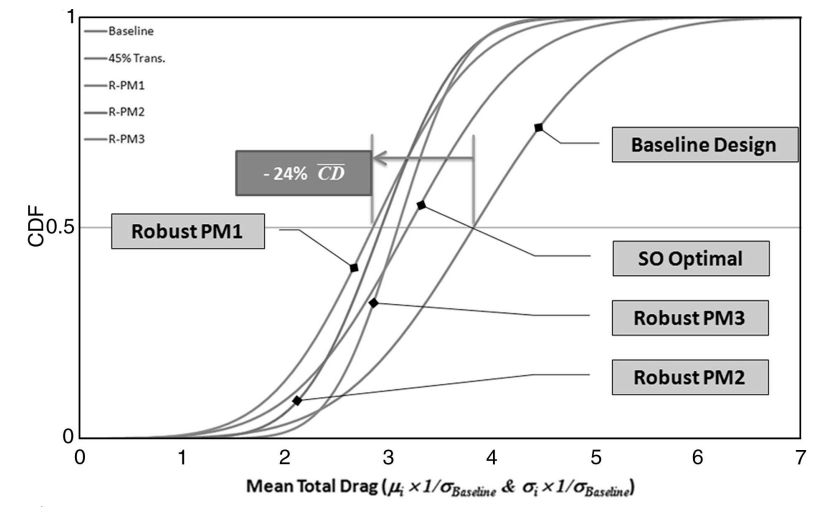

a)
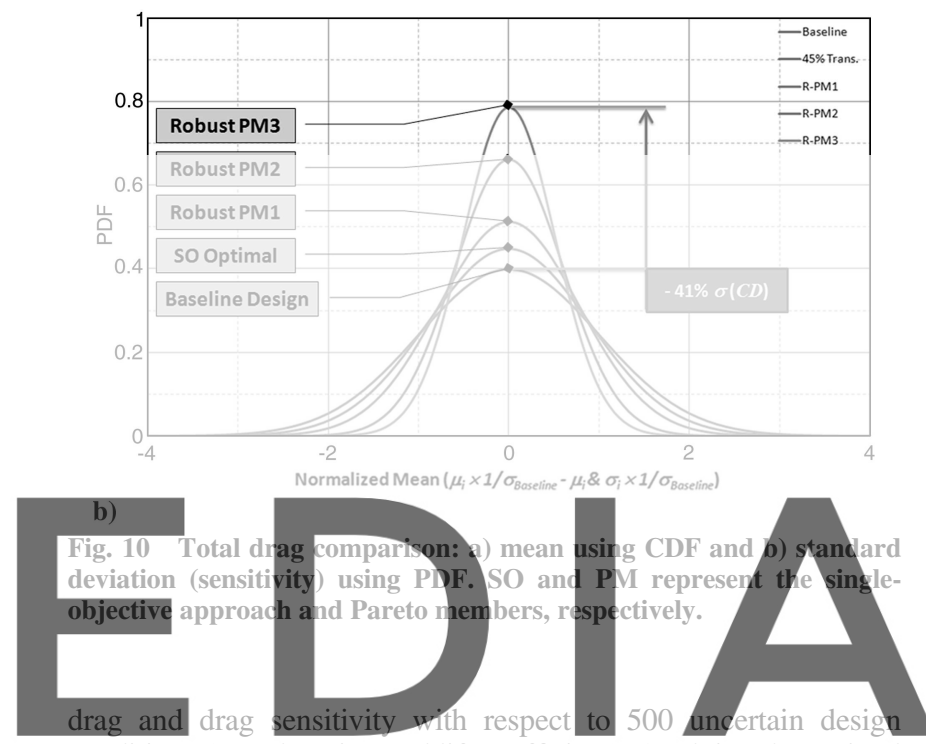

conditions (BLT location and lift coefficient). Applying the optimal SCB obtained by Pareto member 1 produces $26 \%$ lower total drag

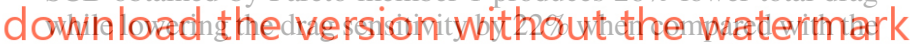
baseline design. The sensitivity obtained by the baseline design and all solutions is more than 2.5 times lower due to the increment of the number of uncertainty design conditions from 6 to 500 . In addition, Fig. 11 compares mean and standard deviation using CDF and PDF.

One thing can be noticed is that $\overline{C d_{\text {Total }}}$ and $\sigma C d_{\text {Total }}$ behaviors obtained by the CDF and PDF (shown in Fig. 10) considering six uncertain flight conditions are similar to the $\overline{C d_{\text {Total }}}$ and $\sigma C d_{\text {Total }}$ obtained by the CDF and PDF (shown in Fig. 11) considering 500 flight conditions between the baseline design and Pareto members 1 to 3 . In other words, the simplified robust method with six uncertain flight conditions still produces both lower total mean drag and drag sensitivity with respect to the variability of BLT positions and lift coefficient.

The design parameters of the SCB obtained by Pareto members 13 are shown in Table 7. It can be seen that the length and height of SCB are reduced by approximately $5 \% c$ and $0.4 \% c$, respectively, when compared with the optimal solution from Sec. V.A (Table 3). Figure 12 compares the geometry of the baseline design and the baseline with optimal SCBs. The baseline (RAE 5243) design with

Table 5 Comparison of fitness values obtained by robust design approach

\begin{tabular}{lccc}
\hline \hline Aerofoil & $\overline{C d_{\text {Total }}}$ & $\sigma C d_{\text {Total }}$ & $\overline{L / D}$ \\
\hline Baseline & 0.00935 & 0.00245 & 87.70 \\
Optimal solution (Sec. V.A) & $0.00780(-17 \%)$ & $0.00219(-11 \%)$ & $105.13(+20 \%)$ \\
with Pareto member 1/SCB & $0.00709(-24 \%)$ & $0.00185(-24 \%)$ & $115.66(+32 \%)$ \\
with Pareto member 2/SCB & $0.00728(-22 \%)$ & $0.00158(-35 \%)$ & $112.64(+28 \%)$ \\
with Pareto member 3/SCB & $0.00764(-18 \%)$ & $0.00145(-41 \%)$ & $107.33(+22 \%)$ \\
\hline \hline
\end{tabular}


Table 6 Comparison of mean and standard deviation of drag obtained by baseline design, single-objective optimal solution, and Pareto members 1 to 3, considering 500 uncertainty flight conditions

\begin{tabular}{lccc}
\hline \hline Aerofoil & $\overline{C d_{\text {Total }}}$ & $\sigma C d_{\text {Total }}$ & $\overline{L / D}$ \\
\hline Baseline & 0.00919 & 0.000893 & 83.31 \\
Optimal solution (Sec. V.A) & $0.00705(-23 \%)$ & $0.000756(-15 \%)$ & $109.59(+31 \%)$ \\
Pareto member 1/SCB & $0.00678(-26 \%)$ & $0.000696(-22 \%)$ & $113.17(+36 \%)$ \\
Pareto member 2/SCB & $0.00720(-22 \%)$ & $0.000591(-34 \%)$ & $106.38(+28 \%)$ \\
Pareto member 3/SCB & $0.00757(-18 \%)$ & $0.000498(-44 \%)$ & $100.93(+21 \%)$ \\
\hline \hline
\end{tabular}

SCB obtained by Pareto member 1 has a maximum thickness of 0.14 $\left(t / c_{\max }=0.14\right)$ at $41 \% c$ from the leading edge and a maximum camber 0.0205 at $62.5 \%$. Adding the SCB obtained by Pareto member 1 increases the maximum camber by 0.002 , and its position is moved toward to the trailing edge by $8.5 \% \mathrm{c}$ while keeping the same maximum thickness as the baseline design.

Figures $13 \mathrm{a}$ and $13 \mathrm{~b}$ show the pressure contour and distribution obtained by the baseline design and Pareto member 1 at $45 \% c$ BLT position. It can be seen that the strong shock on the baseline design shown in Fig. 3 is $77 \%$ weaker by adding SCB on the suction side. The SCB for Pareto member 1 reduces the total drag by $35 \%$ and improves the lift-to-drag ratio by $53 \%$. In addition, the shock (Fig. 3 ) is now moved toward the trailing edge by $2.0 \% \mathrm{c}$.

Figure 14a compares the total drag $C d_{\text {Total }}$ distributions obtained by the baseline design, the optimal solution obtained in Sec. V.A, and Pareto members 1-4. The flight conditions are $M_{\infty} \in[0.6: 0.72]$ with $C_{\text {lFixed }}=0.82, R e=19.0 \times 10^{6}$, and the BLT position at $45 \% \mathrm{c}$. It can be seen that all solution obtained by the single-objective and robust design methods have lower total drag when the Mach number is higher than 0.67 . The optimal solut
lower total drag $(-40 \%)$ at Mach
Pareto members 1 and 4 reduce the total drag
Magh is lower than 0.6775 , while Paret
arag yhen Mach is higher than 0.685 .
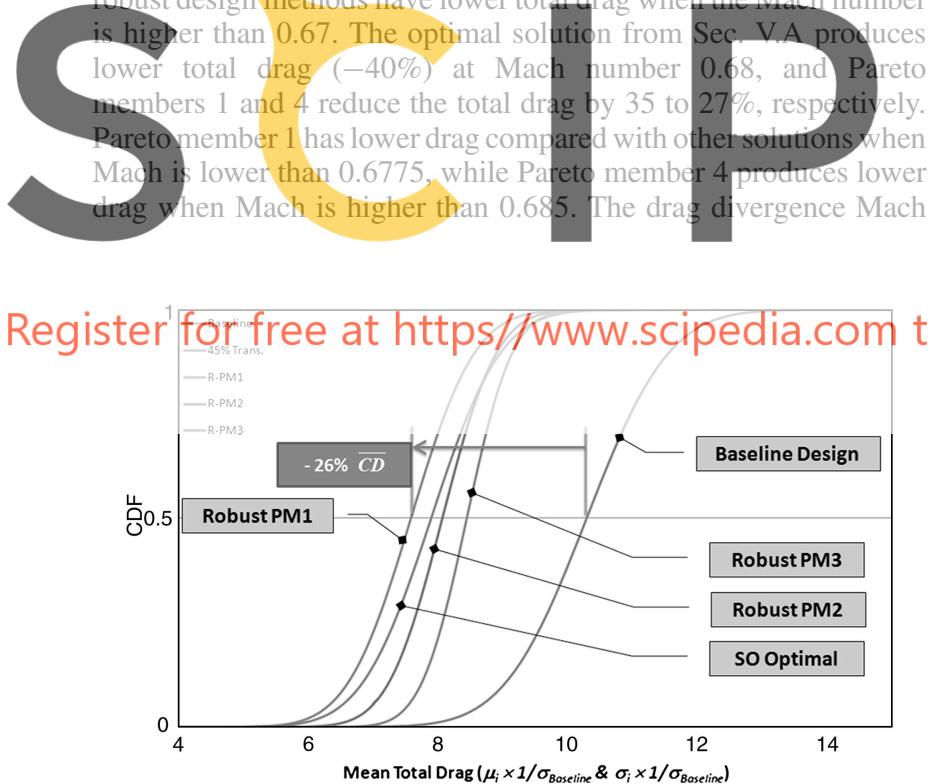

a)

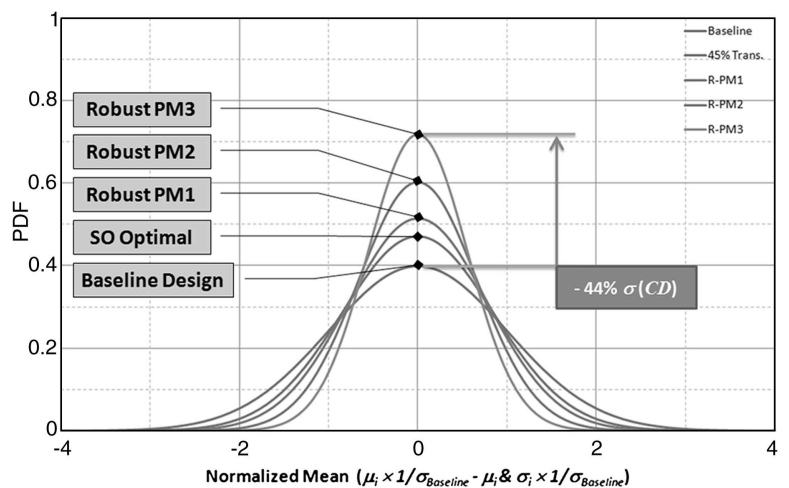

b)

Fig. 11 Total drag comparison with 500 uncertainty flight conditions: a) mean using CDF and b) standard deviation (sensitivity) using PDF.
Table 7 Pareto optimal SCB design components

\begin{tabular}{cccc}
\hline \hline Variables & $\mathrm{SCB}_{L}, \% c$ & $\mathrm{SCB}_{H} \% c$ & $\mathrm{SCB}_{P}\left(\% \mathrm{SCB}_{L}\right)$ \\
\hline Pareto member 1/SCB & 25.93 & 0.76 & 69.3 \\
Pareto member 2/SCB & 27.12 & 0.85 & 76.2 \\
Pareto member 3/SCB & 26.45 & 0.75 & 81.3 \\
\hline \hline
\end{tabular}

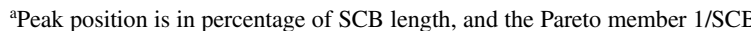
starts from $(x=0.4490, y=0.0859)$ to $(x=0.7083, y=0.0540)$

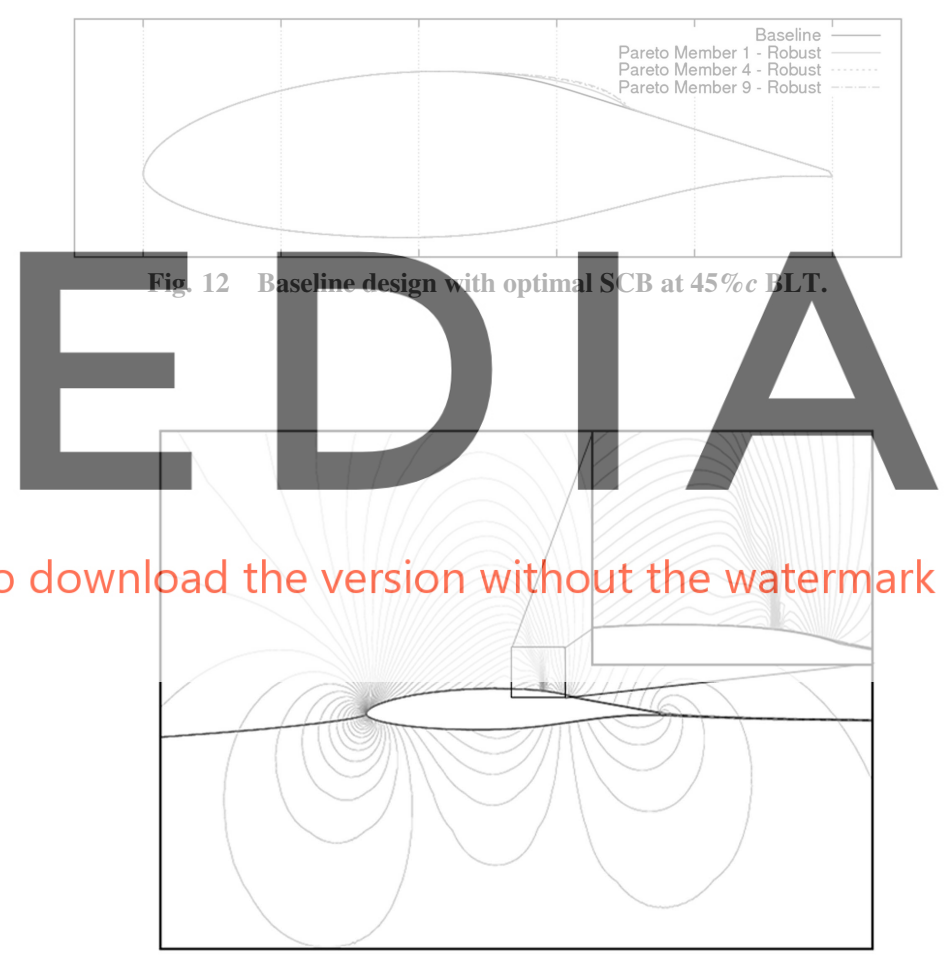

a)

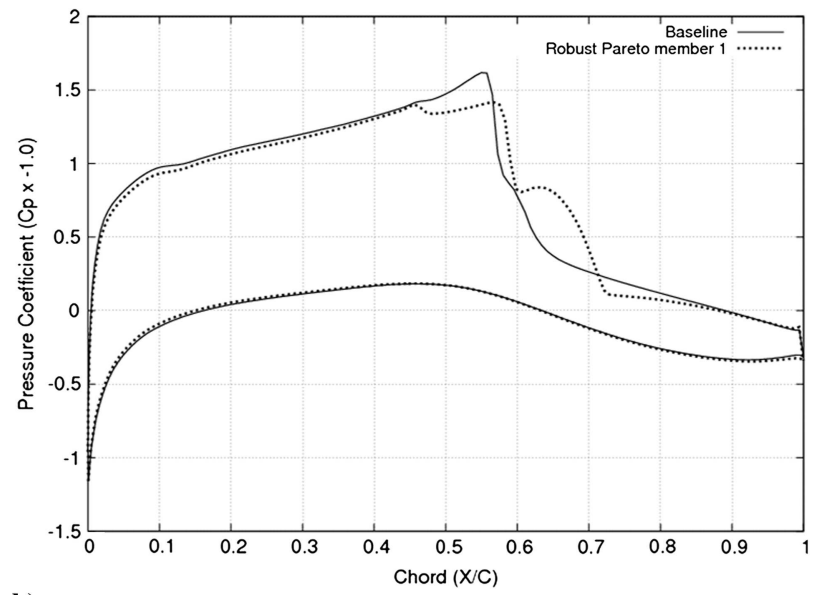

b)

Fig. $13 C_{p}$ contour: a) $C_{p}$ distribution and b) obtained by RAE 5243 with Pareto member 1/SCB at $45 \% c$ BLT position. 
number for the baseline design $\left(M_{c}=0.66\right)$ is extended to 0.69 by applying the optimal SCB obtained by the single-objective and robust design methods.

Figure 14b compares total drag $C d_{\text {Total }}$ distributions at the flow conditions $C_{l} \in[0.1: 1.1]$ with $M_{\text {Fixed }}=0.68, R e=19.0 \times 10^{6}$, and the BLT position at $45 \% c$. Even though the optimal solution obtained by the single-objective method produces lower total drag, Pareto members 1-4 have a stable total drag distribution at $C_{l}$ range [0.6:0.82] without fluctuation due to the stable wave drag. The drag divergence $C_{l}$ number for the baseline design $\left(C_{l c}=0.5\right)$ is extended to $0.8-0.9$ by applying the optimal SCB obtained by the singleobjective and robust design methods.

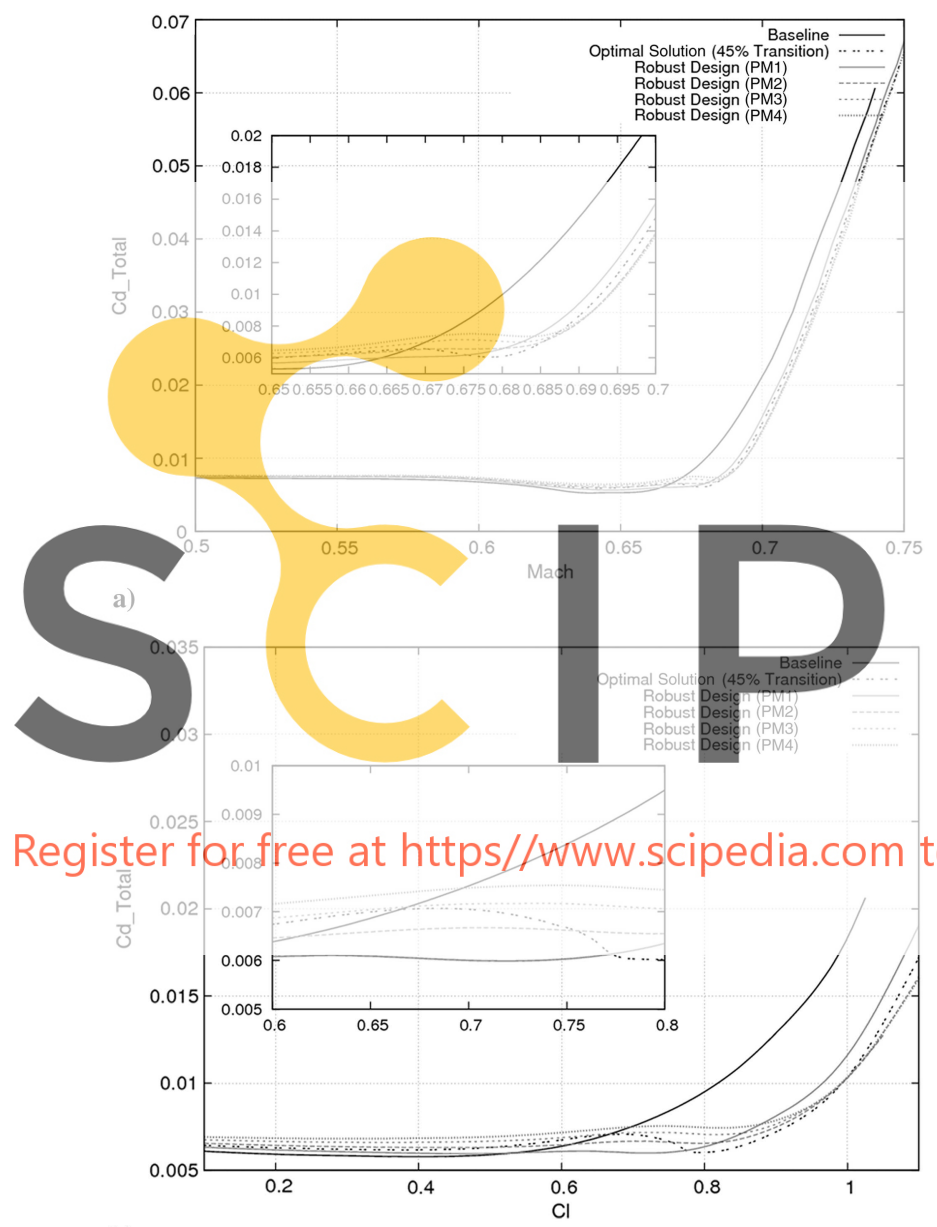

b)

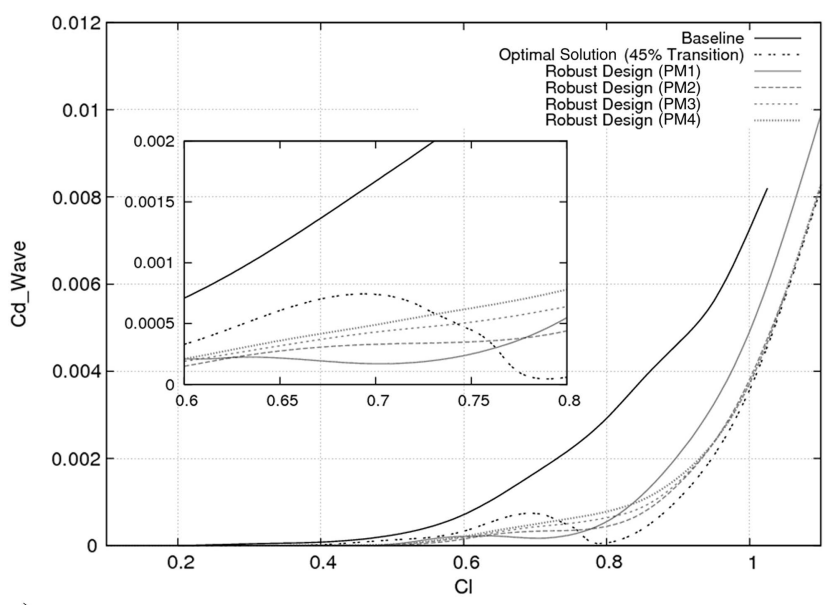

c)

Fig. 14 Comparisons at $45 \%$ c BLT position. PM denotes Pareto member.
Figure 14c compares wave drag $C d_{\text {Wave }}$ distributions obtained by the baseline design and single-objective and robust design methods. It can be seen that Pareto members 1-4 produce a stable wave drag when compared with the baseline design and the optimal solution obtained by the single-objective approach.

Figure 15a compares the total drag distributions at a range of Mach numbers; that is, $M_{\infty} \in[0.5: 0.75]$ with $C_{l \text { Fixed }}=0.82, R e=19.0 \times$ $10^{6}$, and the BLT position at $25 \% \mathrm{c}$. The optimal solution obtained in Sec. V.A and Pareto members 1-4 produce lower total drag compared with the baseline design when the Mach number is higher than 0.665. The optimal solution (Sec. V.A), Pareto members 1 and 4 reduce the total drag by 25,24 , and $23 \%$, respectively, when compared with the

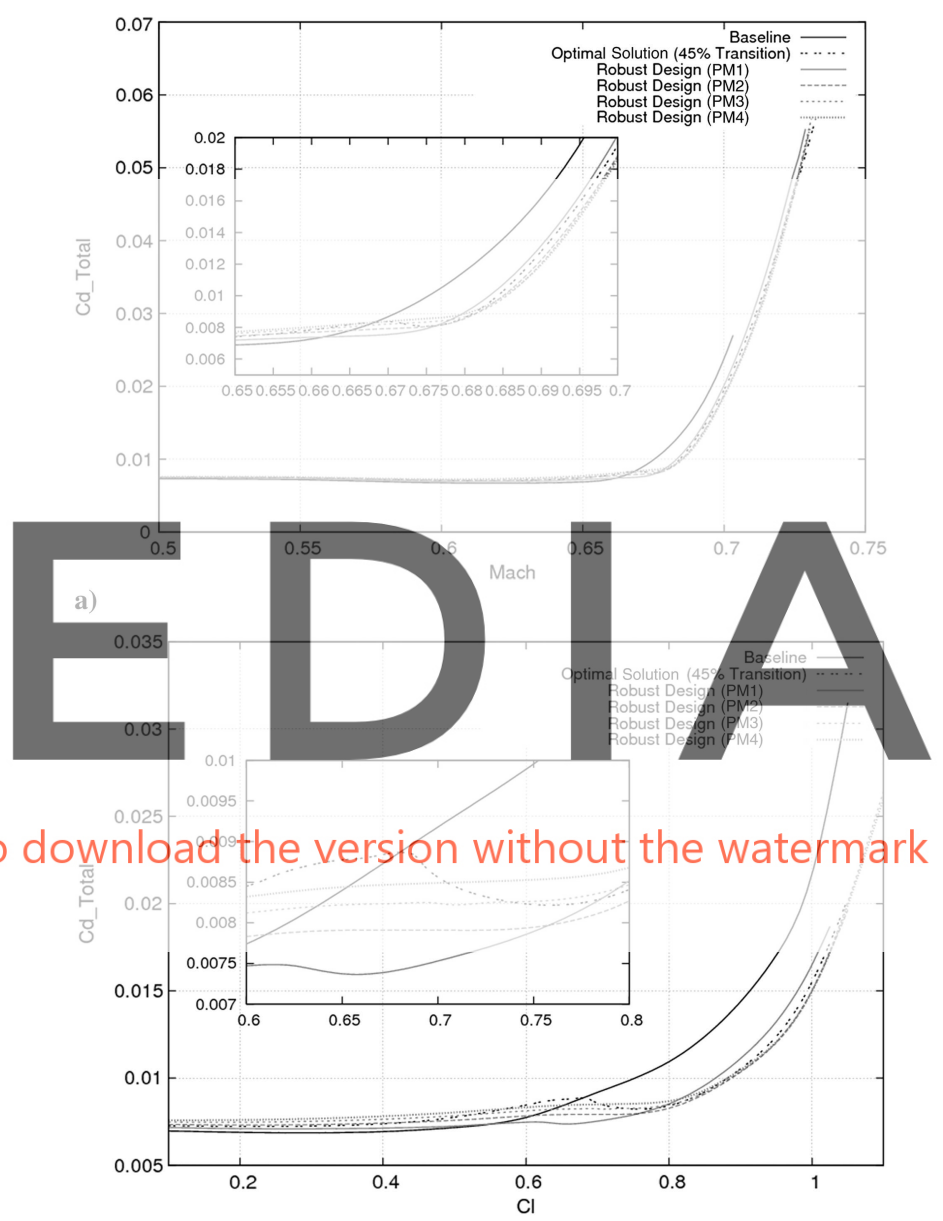

b)

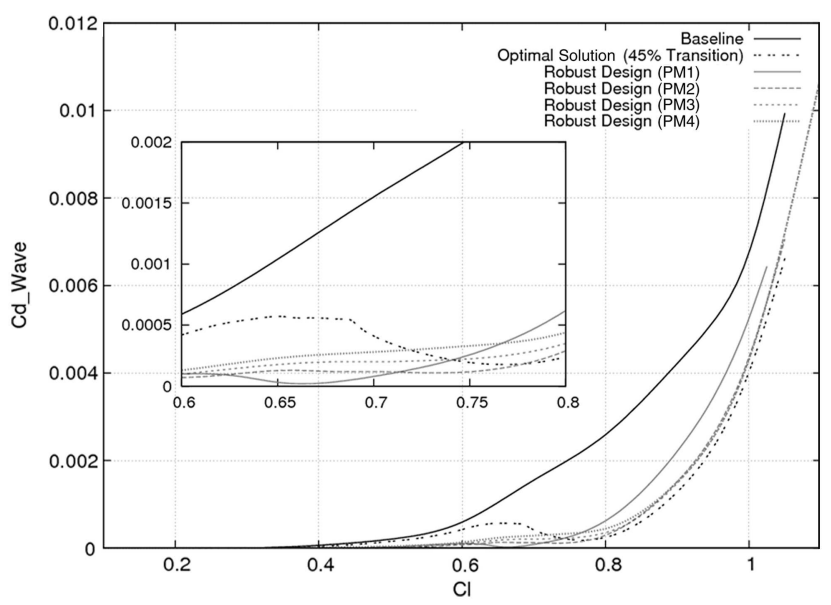

c)

Fig. 15 Comparisons at 25\%c BLT position. PM denotes Pareto member. 
Table 8 Six flight conditions

\begin{tabular}{cccccc}
\hline \hline Conditions & $M_{\infty}$ & $R e$ & $C_{l}$ & $x_{\text {Trans }}-$ Upper & $x_{\text {Trans }}-$ Lower \\
\hline Flight condition 1 & 0.69 & $11.7 \times 10^{6}$ & 0.54 & 0.51 & 0.54 \\
Flight condition 2 & 0.69 & $11.7 \times 10^{6}$ & 0.69 & 0.51 & 0.54 \\
Flight condition 3 & 0.70 & $11.7 \times 10^{6}$ & 0.38 & 0.51 & 0.54 \\
Flight condition 4 & 0.70 & $11.7 \times 10^{6}$ & 0.50 & 0.51 & 0.54 \\
Flight condition 5 & 0.73 & $11.7 \times 10^{6}$ & 0.22 & 0.51 & 0.54 \\
Flight condition 6 & 0.73 & $11.7 \times 10^{6}$ & 0.34 & 0.51 & 0.54 \\
\hline \hline
\end{tabular}

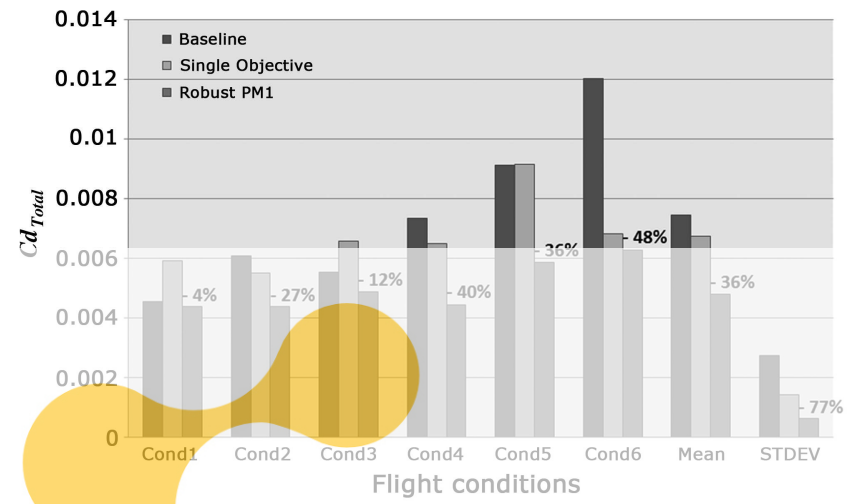

Fig. 16 Drag reduction obtained by the baseline design, the singleobjective solution, and robust Pareto member 1 (note that robust PM1

$$
\text { Re }
$$

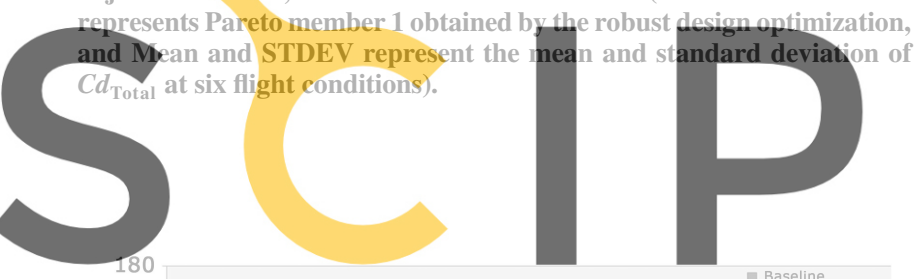

$160+40 \% \quad$ Baseline

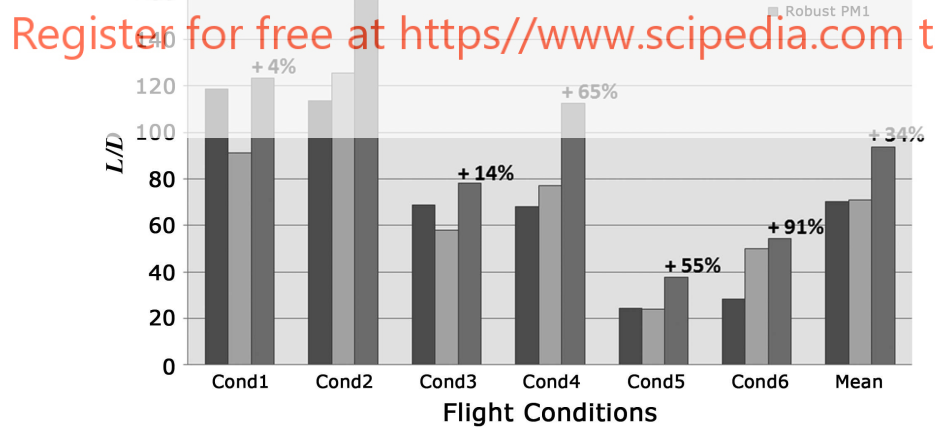

Fig. 17 Drag reduction obtained by the baseline design, the singleobjective solution, and robust Pareto member 1. baseline design. Pareto member 1 has lower drag when Mach is lower than 0.6775 , while Pareto member 4 has lower drag when Mach is lower than 0.685 . The drag divergence Mach number for the baseline design $\left(M_{c}=0.66\right)$ is extended to 0.685 by applying the optimal SCB obtained by the single-objective and robust design methods.

Figure $15 \mathrm{~b}$ compares total drag distributions at a range of $C_{l}$; that is, $C_{l} \in[0.1: 1.1]$ with $M_{\text {Fixed }}=0.68, R e=19.0 \times 10^{6}$, and the BLT at $25 \% \mathrm{c}$. The optimal solution from Sec. V.A fluctuates at the range of $C_{l}=$ [0.6:0.82], while Pareto members 1 and 4 have a stable $C_{l}$ distribution. The drag divergence $C_{l}$ number for the baseline design $\left(C_{l c}=0.6\right)$ is extended to 0.8 by applying the optimal SCB obtained by the single-objective and robust design methods.

Figure $15 \mathrm{c}$ compares wave drag $C d_{\text {Wave }}$ distributions obtained by the baseline design and the single-objective and robust design methods. It can be clearly seen that Pareto members $1-4$ produce stable wave drag when compared with the baseline design and singleobjective approach. It should be noticed that the critical lift coefficient numbers $C_{l c}$ for the baseline design, the optimal solution obtained by the single-objective approach, and the Pareto members obtained by the robust design method are 0.3, 0.4, and 0.5, respectively,

The baseline design with the optimal SCB obtained by the singleobjective and robust design methods are also tested at six normal flight conditions, shown in Table 8 .

The histogram shown in Fig. 16 compares the total drag obtained by the baseline design and the single-objective (Sec. V.A) and robust design approaches. It can be seen that the baseline design with the optimal SCB obtained by the robust design (denoted as robust MP1) produces lower total drag when compared with the baseline design

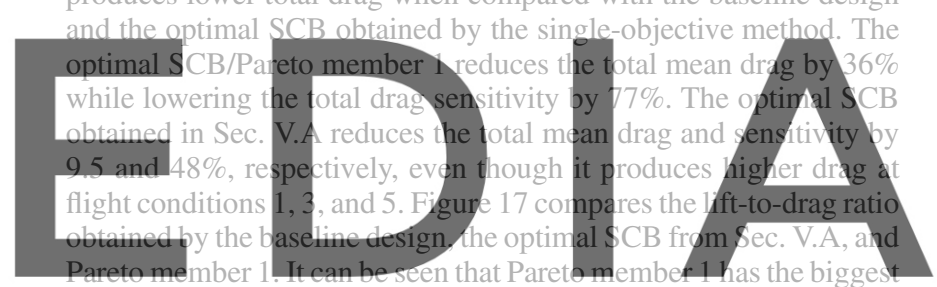

lift-to-drag improvement at flight condition 4. The pressure coefficient distributions at six flight conditions are shown in Figs. A1-

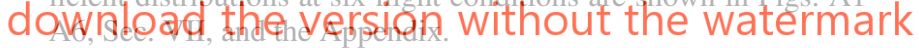

One example (Cond4 from Fig. 17) is shown in Fig. 18, where the pressure contours obtained by the baseline design and Pareto member 1 are illustrated. Even though the SCB obtained by Pareto member 1 is optimized at the critical flight condition, the optimal $\mathrm{SCB} /$ Pareto member 1 reduces the wave drag $\left(C d_{\text {Wave }}=0.0025\right)$ obtained by the baseline design by $99.5 \%\left(C d_{\text {Wave }}=0.00001\right)$ and reduces the total drag by $40 \%$, which leads to $65 \%$ improvement of $L / D$ when compared with the baseline design.

To summarize the design test cases (Secs. V.A and V.B), the design engineer can choose one of the solutions (Pareto members 1 to 4) obtained by the robust multiobjective design optimization due to two main reasons. The first is that, even though the optimal solution from Sec. V.A produces lower total drag at the standard/mean flight conditions (BLT position at 45\%c), Pareto members 1 to 4 have lower sensitivity at the variability of $C_{l}$ and BLT positions $[25 \% c: 50 \% c]$. In addition, it is clearly shown that applying SCB

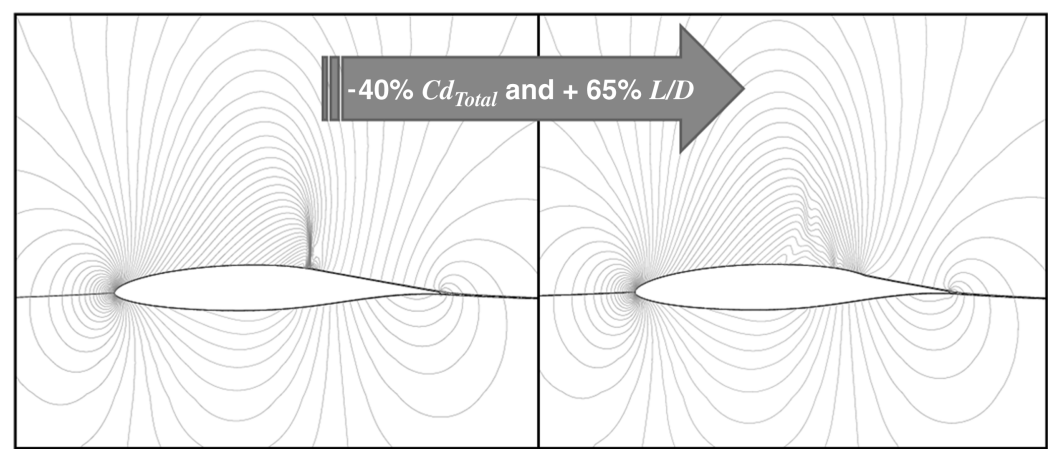

Fig. $18 C_{p}$ contour comparison obtained by the baseline design (left) and Pareto member 1 (right) at flight condition 4. 
obtained by the robust design method stabilizes the total drag of the baseline design at both normal and critical flight conditions. The second is that Pareto members 1 to 4 have a smaller SCB than the optimal solution obtained by the single-objective method. In other words, the manufacturer can save material and weight costs, and fewer modifications are needed to the manufacturing system.

\section{Discussion}

This paper explored the practical applications of MOEA with uncertainty for SCB shape optimization. The results from two test cases raise two discussion points and possible research avenues:

1) In this paper, a simplified robust design technique considering only six uncertainty flight conditions is used, and the obtained optimal solutions produce very similar statistical (CDF and PDF) behavior when compared with the CDF and PDF curves obtained by considering the 500 uncertainty flight conditions. In other words, even though it is desirable to use a detailed robust design technique with more than 100 samplings for uncertainties conditions, this simplified robust method can be applied to a preliminary robust design optimization with low computational cost to find a set of designs that have higher mean performance and lower sensitivity. As ongoing research focuses on applying the concept of hierarchical asynchronous approach for not only using low/middle flow solvers but also using detailed/intermediate/preliminary robust design methods to save a computational cost.

2) The first discussion point on SCB design variation is that the algorithm considers only three control points, which may not be enough to capture the best SCB geometry. Therefore, more than three control points need to be considered for a detailed SCB design

R
optimization. The second point is that the two test cases considered aerodynamic performance only, without any structural aspects. Even though SCB produces significant dra unstable structure. The current work (aerostructure) design optimization in

VH. Conclusions

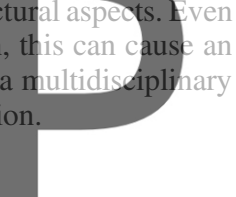

In this paper, the robust evolutionary optimization technique has

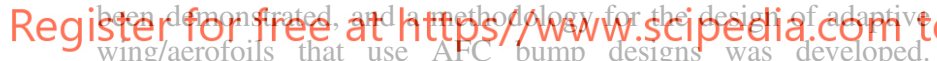
Analytical research shows the benefits of the coupling optimization method with robust design techniques to produce stable and high performance solutions. The use of SCB on an existing aerofoil can reduce significant transonic drag, which will save operating and manufacturing costs as well as emission reduction. Future work will focus on robust design optimization of SCB on a three-dimensional wing.

\section{Appendix}

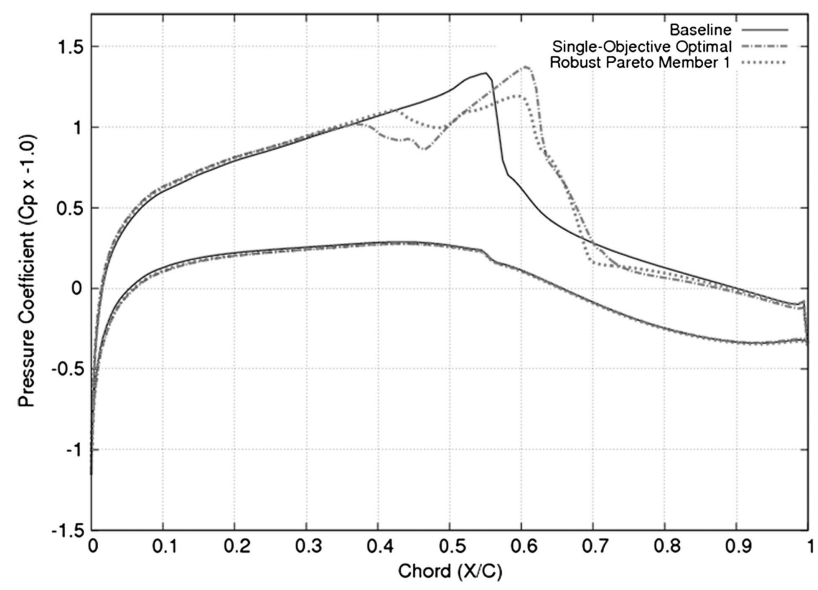

Fig. A1 $C_{p}$ distribution comparison at flight condition 1 (shown in Table 8).

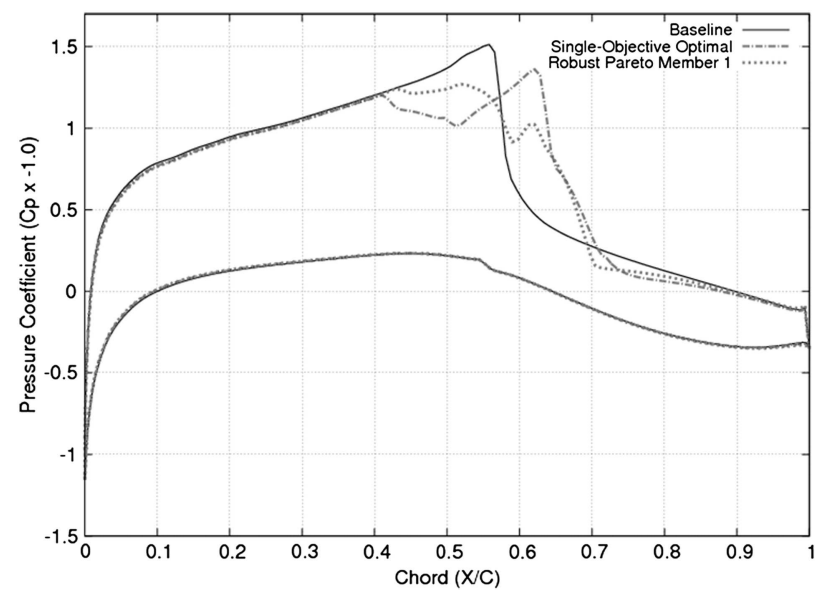

Fig. A2 $C_{p}$ distribution comparison at flight condition 2 (shown in Table 8).

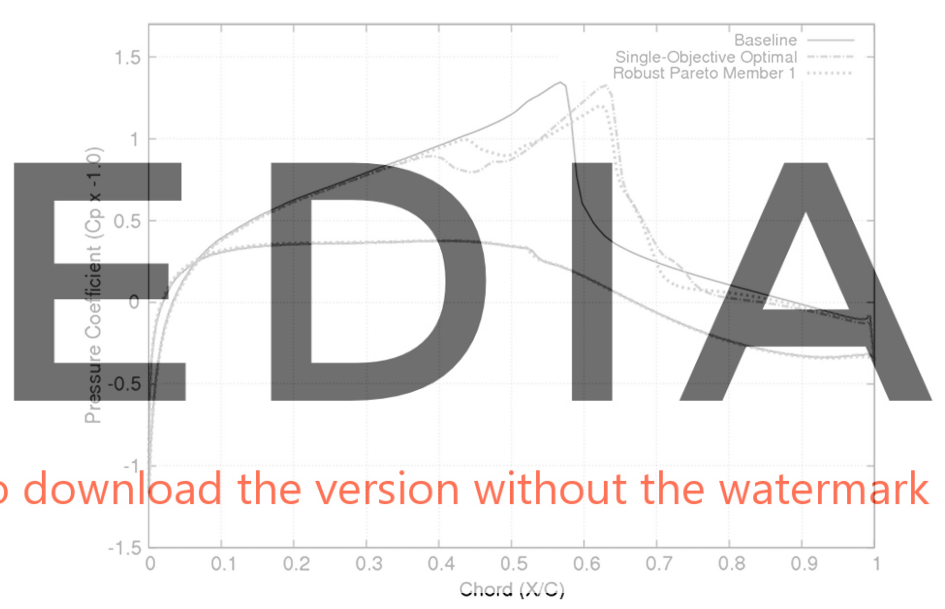

Fig. A3 $C_{p}$ distribution comparison at flight condition 3 (shown in Table 8).

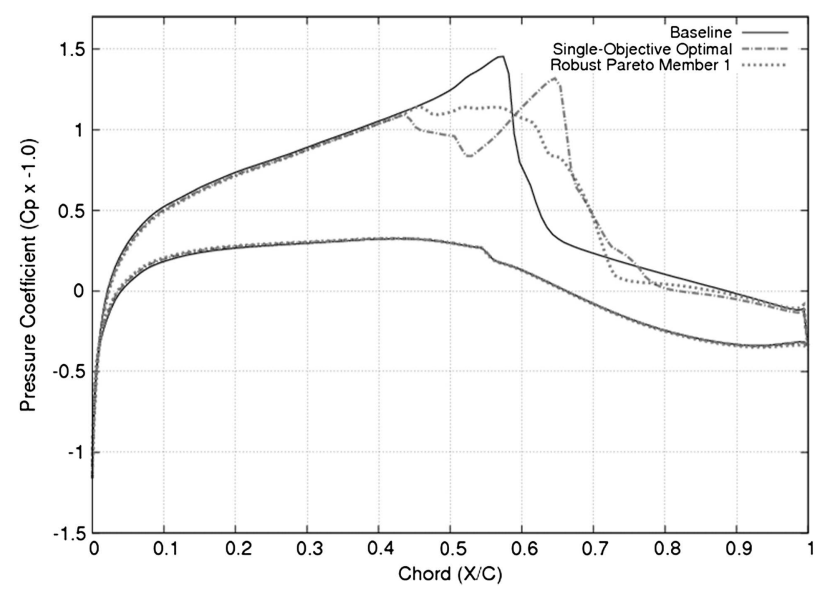

Fig. A4 $C_{p}$ distribution comparison at flight condition 4 (shown in Table 8). 


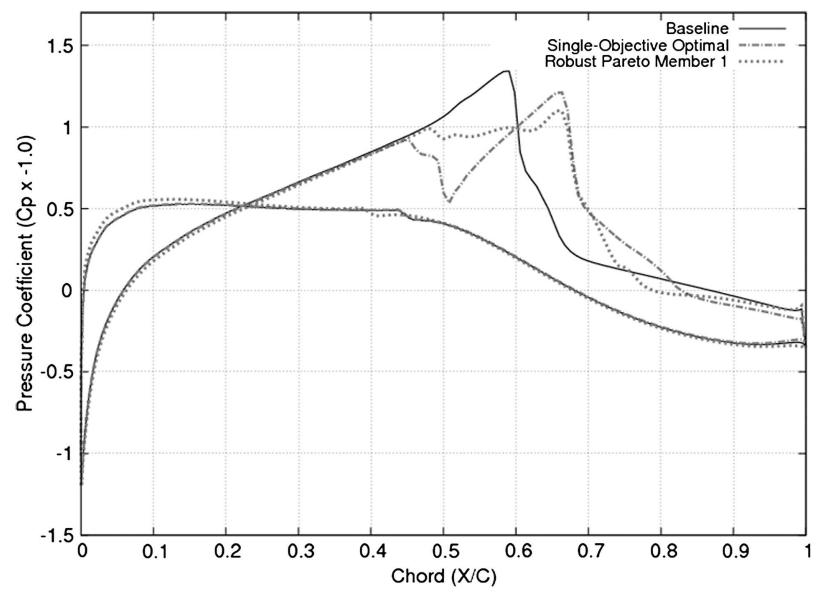

Fig. A5 $C_{p}$ distribution comparison at flight condition 5 (shown in Table 8).

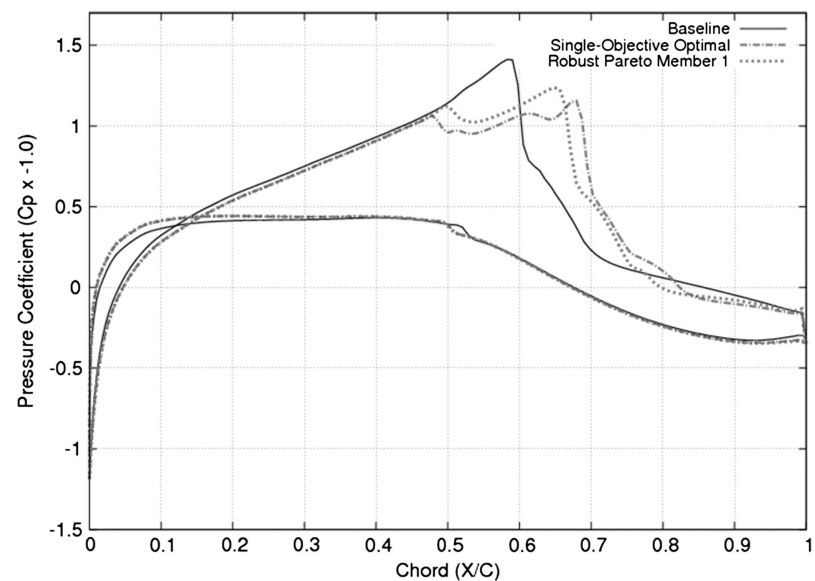

Fig. A6 $C_{p}$ distribution comparison at flight condition 6 (shown in Table 8).

\section{Acknowledgments}

The authors gratefully acknowledge E. J. Whitney and M. Sefrioui of Dassault Aviation for fruitful discussions on hierarchical evolutionary algorithms and their contribution to the optimization procedure. The authors also acknowledge M. Drela at the Massachusetts Institute of Technology for providing MSES software. The authors are thankful to the organizers of the database workshop event "Integrated Multiphysics Simulation and Design Optimization: An Open Database Workshop for Multiphysics Software Validation", ${ }^{* *}$ which took place at the University of Jyvaskyla, Finland, 10-13 March 2010, igniting fruitful discussions on active flow control techniques in computational fluid dynamics design with contributors to the bump test case problem.

\section{References}

[1] Lee, D. S., Gonzalez, L. F., Srinivas, K., and Periaux, J., "Robust Evolutionary Algorithms for UAV/UCAV Aerodynamic and RCS Design Optimisation," Computers and Fluids, Vol. 37, No. 5, 2008, pp. $547-564$

doi:10.1016/j.compfluid.2007.07.008
[2] Lee, D. S., Gonzalez, L. F., Srinivas, K., and Periaux, J., "Robust Design Optimisation Using Multi-Objective Evolutionary Algorithms," Computers and Fluids, Vol. 37, No. 5, 2008, pp. 565-583. doi:10.1016/j.compfluid.2007.07.011

[3] Taguchi, G., and Chowdhury, S., Robust Engineering, McGraw-Hill, New York, 2000.

[4] Lee, D. S., Gonzalez, L. F., Periaux, J., and Srinivas, K., "Evolutionary Optimisation Methods with Uncertainty for Modern Multidisciplinary Design in Aeronautical Engineering," 100 Volumes of Notes on Numerical Fluid Mechanics: 40 Years Numerical Fluid Mechanics, Springer, Berlin, 2009, pp. 271-284.

[5] Ashill, P. R., Fulker, L. J., and Shires, A., "A Novel Technique for Controlling Shock Strength of Laminar-Flow Aerofoil Sections," Proceedings 1st European Forum on Laminar Flow Technology, Hamburg, Defence Research Agency, Farnborough, England, U.K., March 1992, pp. 175-183.

[6] Qin, N., Zhu, Y., and Shaw, S. T., "Numerical Study of Active Shock Control for Transonic aerodynamics," International Journal of Numerical Methods for Heat and Fluid Flow, Vol. 14, No. 4, 2004, pp. 444-466. doi:10.1108/09615530410532240

[7] Wong, W. S., Qin, N., Sellars, N., Holden, H., and Babinsky, H., "A Combined Experimental and Numerical Study of Flow Structures over Three-Dimensional Shock Control Bumps," Aerospace Science and Technology, Vol. 12, No. 6, 2008, pp. 436-447. doi:10.1016/j.ast.2007.10.011

[8] Qin, N., Wong, W. S., and LeMoigne, A., "Three-Dimensional Contour Bumps for Transonic Wing Drag Reduction," Proceedings of the Institution of Mechanical Engineers. Part G, Journal of Aerospace Engineering, Vol. 222, No. 5, 2008, pp. 619-629. doi:10.1243/09544100JAERO333

[9] Lee, D. S., Gonzalez, L. F., and Whitney, E. J., Multi-Objective, Multidisciplinary Multi-Fidelity Design Tool: HAPMOEA: User Guide, Univ. of Sydney, Sydney, NSW, Australia, 2007.

[10] Hansen, N., and Ostermeier, A., "Completely Derandomized SelfAdaptation in Evolution Strategies," Evolutionary Computation, Vol. 9, No. 2, 2001, pp. 159-195. doi:10.1162/106365601750190398

[11] Koza, J., Genetic Programming II, Massachusetts Institute of Technology, Cambridge, MA, 1994.

[12] Michalewicz, Z., Genetic Algorithms + Data Structures $=$ Evolution Programs, Springer-Verlag, New York, 1992.

[13] Wakunda, J., and Zell, A., "Median-Selection for Parallel Steady-State Evolution Strategies," Parallel Problem Solving from Nature: PPSN VI, edited by M. Schoenauer, K. Deb, G. Rudolph, X. Yao, E. Lutton, J. Merelo, and H.-P. Schwefel, Springer, Berlin, 2000, pp. 405-414.

[14] Van Veldhuizen, D. A., Zydallis, J. B., and Lamont, G. B., "Considerations in Engineering Parallel Multiobjective Evolutionary Algorithms," IEEE Transactions on Evolutionary Computation, Vol. 7 , No. 2, 2003, pp. 144-173. doi:10.1109/TEVC.2003.810751

[15] Sefrioui, M., and Périaux, J., "A Hierarchical Genetic Algorithm Using Multiple Models for Optimization," Parallel Problem Solving from Nature, PPSN VI, edited by M. Schoenauer, K. Deb, G. Rudolph, X. Yao, E. Lutton, J. J. Merelo, and H.-P. Schwefel, Springer, New York, 2000, pp. 879-888.

[16] Drela, M., A User's Guide to MSES 2.95, MIT Computational Aerospace Sciences Lab., Cambridge, MA, Sept. 1996.

[17] Lee, D. S., Periaux, J., Pons-Prats, J., Bugeda, G., and Onate, E., "Double Shock Control Bump Design Optimisation Using Hybridised Evolutionary Algorithms," Special Session (SO35-IEEE CEC): Evolutionary Computation in Aerospace Sciences, 2010 IEEE World Congress On Computational Intelligence (WCCI 2010), Barcelona, July 2010. doi:10.1109/CEC.2010.5586379

[18] Iman, R. L., Davenport, J. M., and Zigler, D. K., Latin Hypercube Sampling (Program User's Guide), Office of Scientific and Technical Information Rept. 5571631, Washington, D.C., 1980. 